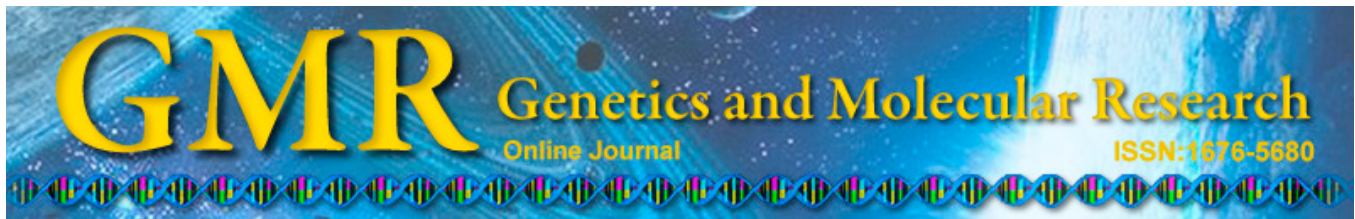

\title{
Association between $P A R K 16$ gene polymorphisms and susceptibility of Parkinson's disease in a Chinese population
}

\author{
H. Xia ${ }^{1 *}$, Q. Luo ${ }^{1 *}$, X.X. Li ${ }^{2}$ and X.L. Yang ${ }^{1}$ \\ ${ }^{1}$ Cadre's Wards, The Affiliated Tumor Hospital of Xinjiang Medical University, \\ Urumqi, Xinjiang, China \\ 2Internal Medicine-Neurology, Leshan People's Hospital, Leishan, \\ Sichuan, China \\ *These authors contributed equally to this study. \\ Corresponding author: X.L. Yang \\ E-mail: poplar862@sohu.com
}

Genet. Mol. Res. 14 (2): 2978-2985 (2015)

Received March 19, 2014

Accepted October 7, 2014

Published April 10, 2015

DOI http://dx.doi.org/10.4238/2015.April.10.7

ABSTRACT. Recent genome-wide association studies identified 11
risk loci in different populations of familial and sporadic Parkinson's
disease (PD) patients. Few loci have been verified in different European
and Asian populations. We also validated 2 new single-nucleotide
polymorphisms, rs 947211 and rs 823144 , in PARK16 to explore their
association with susceptibility to PD in the Xinjiang Uygur and
Han populations. This case-control study included 312 PD patients
(130 Uygur and 182 Han) and 359 control subjects ( 179 Uygur and
180 Han). Polymerase chain reaction-restriction fragment length
polymorphism analysis and DNA sequencing were used to detect the
rs947211 and rs 823144 polymorphism in the PARK16 gene between the
Xinjiang Uygur and Han populations. Frequencies of the A allele and
AA genotype ( 42.1 and $15.7 \%$, respectively) of rs 947211 in PD patients
were significantly lower than those in the control group ( 54.7 and
$28.7 \%$, respectively, P $<0.01$ ). A allele and AA genotype frequencies of 
rs 823144 were 56.8 and $31.8 \%$ in the PD patients group and were 54.1 and $29.3 \%$ in the control group; no significant difference was found $(\mathrm{P}$ $>0.05$ ). In both the Han and Uygur groups, the rs 947211 polymorphism was associated with PD. Haplotype analysis also indicated that the A-A and G-A haplotypes were associated with PD. We found that the rs947211 polymorphism may be a susceptibility marker for PD in the Chinese population; the A-A and G-A haplotypes may be a protective factor and a risk factor, respectively, for PD in the Chinese population.

Key words: Genetic polymorphism; Parkinson's disease; PARK16

\section{INTRODUCTION}

Parkinson's disease (PD) is a complex neurodegenerative disease; the main pathological changes include depigmentation of substantia nigra dopaminergic neurons and formation of Lewy bodies. Most PD patients are sporadic and exhibit no clear genetic history (Lang and Lozano, 1998; Goedert et al., 2012). Some genetic variants appear to increase the susceptibility to sporadic PD in the general population. Recent genome-wide association studies conducted in North America, Europe, and Asia showed that the PARK16 gene polymorphism was associated with PD (Satake et al., 2009; Simón-Sánchez et al., 2011; Liu et al., 2011).

PARK16 (OMIM 613164) is located at chromosome 1q32, which includes the transcribed regions of 5 related genes: RAB7L1, SLC41A1, NUCKS1, SLA45A3, and PM20D1. The SLC41A1, NUCKS1, and RAB7L1 genes were shown to be associated with PD (Tucci et al., 2010). However, studies in Asia, including China, Singapore, and Taiwan, have shown inconsistent results. Furthermore, additional studies have shown that genetic polymorphisms in PARK16 have significant regional and ethnic differences (Tan et al., 2010; Vilariño-Guell et al., 2010; Chang et al., 2011; Yan et al., 2011; Chiang et al., 2012). In this study, we selected Uygur and Han ethnic patients with PD and healthy controls in Xinjiang to explore the relationship between the PARK16 gene rs949211 and rs823144 polymorphisms and PD.

\section{MATERIAL AND METHODS}

\section{Subjects and methods}

The PD patient and control groups were selected from the First Affiliated Hospital of Xinjiang Medical University from June 2010 to April 2013. A total of 312 PD patients (all sporadic) who visited the neurology specialist clinic were enrolled. Diagnosis was based on the PD diagnostic criteria of the UK Brain Bank. Exclusion criteria were as follows: Parkinson's syndrome caused by cerebrovascular disease, encephalitis, trauma, drugs, Parkinson's plus syndrome, and severe systemic disease. A total of 359 volunteers from the same area with no family history of PD and PD clinical manifestations were included as the control group. Gender, age, and ethnicity were matched between the 2 groups. The study was approved by the First Affiliated Hospital of Xinjiang Medical Ethics Committee, and all subjects signed informed consent. 


\section{DNA extraction}

First, $2 \mathrm{~mL}$ fasting blood was acquired from all subjects. Next, using ethylenediaminetetraacetic acid as an anticoagulant, genomic DNA was extracted using a kit (Shanghai Tiangen Bio Co., Ltd., Shanghai, China).

\section{Design of primers and amplification of the target gene}

According to previous genome-wide association studies (Satake et al., 2009) and the SNP information for the PARK16 gene in National Center for Biotechnology Information SNP database (NCBI, http://www.ncbi.nlm.nih.gov/SNP), we chose rs947211 and rs823144. Primers were designed based on the DNA sequences (Table 1). For DNA amplification, the total polymerase chain reaction (PCR) volume was $20 \mu \mathrm{L}$, including $0.5 \mu \mathrm{L} 100 \mathrm{ng} / \mu \mathrm{L}$ upstream and downstream primers, $10 \mu \mathrm{L} 2 \mathrm{X}$ Power Taqman Master Mix (Beijing Baitaike

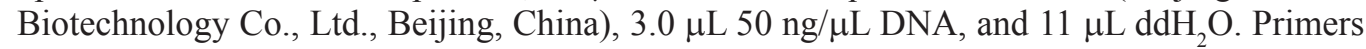
were synthesized by Sangon Shanghai Co., Ltd. (Shanghai, China). Each sample was amplified for 35 cycles in a 9700 amplification system (Applied Biosystems, Foster City, CA, USA).

\begin{tabular}{|c|c|c|c|}
\hline SNPs & Primers & Products (bp) & Annealing temperatures $\left({ }^{\circ} \mathrm{C}\right)$ \\
\hline \multirow[t]{2}{*}{ rs947211 } & F: 5'TTTCTGCTTTTCTCACCTCCT3' & \multirow[t]{2}{*}{307} & \multirow[t]{2}{*}{58.7} \\
\hline & R: 5'ATTTGGGCCTGGGAGTATCT3' & & \\
\hline \multirow[t]{2}{*}{ rs823144 } & F: 5'CTGACTGCCGAGAAACTGGAC3' & \multirow[t]{2}{*}{571} & \multirow[t]{2}{*}{53.6} \\
\hline & R: 5'GATGAGGCTTGGGCTTTTACTA3' & & \\
\hline
\end{tabular}

\section{PCR product detection}

For PCR product detection, $7 \mu \mathrm{L}$ PCR products were mixed with $2 \mathrm{X}$ bromo buffer and separated by $4 \%$ agarose gel electrophoresis. Nucleic acid dyes were used to stain the samples after $4 \mathrm{~V} / \mathrm{cm}$ voltage electrophoresis for $1 \mathrm{~h}$. Bands were observed and photographed under ultraviolet light.

\section{Enzyme digestion genotyping}

For genotyping, $8 \mu \mathrm{L}$ PCR products and $2 \mu \mathrm{L} 10 \mathrm{X}$ buffer were combined with $5 \mathrm{U}$ restriction endonucleases $H p y 188 \mathrm{I}$ and $B s a \mathrm{HI}$ (New England Biolabs, Ipswich, MA, USA) and $20 \mu \mathrm{L}$ sterile double-distilled water at $37^{\circ} \mathrm{C}$ overnight $(16 \mathrm{~h})$. Next, $9 \mu \mathrm{L}$ digestion products were separated on a $4 \%$ agarose gel for ethidium bromide staining at $110 \mathrm{~V}$ for $1.5 \mathrm{~h}$. A Gel Doc 1000 gel imaging analysis system (Bio-Rad, Hercules, CA, USA) was used to observe the electrophoretic bands.

\section{Direct sequencing}

To determine the accuracy of our results, we randomly selected $10 \%$ of the samples (31 in the patient group, 36 in the control group) for direct sequencing. 


\section{Statistical analysis}

Genotype frequencies were calculated using the gene-counting method. The HardyWeinberg equilibrium test of genotypes was performed using the Arlequin software. The ages between the 2 groups were compared using the independent sample $t$-test, and gender, allele and genotype frequencies between the 2 groups were compared using the $\chi^{2}$ test. $\mathrm{P}<0.05$ was considered to be statistically significant.

\section{RESULTS}

\section{Hardy-Weinberg equilibrium test}

The $\chi^{2}$ values for the rs947211 and rs823144 genotypes of the PARK16 gene in the case and control groups were 1.97 and 0.78 and 0.099 and 0.002 , respectively, with all $\mathrm{P}>$ 0.05 . The sample population was found to be in Hardy-Weinberg equilibrium and thus represented the whole population.

\section{Enzyme digestion of the PARK16 gene}

As shown in Figures 1 and 2, the PCR products (307 bp) of rs947211 were digested with Hpy188I. The AA genotype showed 1 band (307 bp), the GG genotype showed 2 bands (147 and $160 \mathrm{bp}$ ), and the AG genotype produced 3 bands (307, 147, and $160 \mathrm{bp}$ ). The PCR products of rs 823144 were $571 \mathrm{bp}$; after $B s a \mathrm{HI}$ digestion, the 25 -bp fragment was too small to be observed on the agarose gel. Therefore, the AA genotype had 1 band (546 bp), the CC genotype had 2 bands (383 and 163 bp), and the AC genotype had 3 bands (163, 383, and 546). Direct sequencing verified the genotyping results.

\section{Genotype and allele distribution frequencies of rs947211 and rs823144 in the 2 groups}

As shown in Table 2, the genotype and allele frequencies of the rs947211 polymorphism were compared between the case and control groups; the difference was statistically significant $[\mathrm{P}<0.01$, odds ratio $(\mathrm{OR})=0.60,95 \%$ confidence interval: $0.48-0.76]$. The rs823144 genotype and allele frequencies were not significantly different between the case and control groups $(\mathrm{P}>0.05)$. After ethnicity grouping, the genotype and allele frequencies of the rs947211 were significantly different between the case and control groups, both in the Han and Uygur populations (both $\mathrm{P}<0.05$ ).

\section{Haplotype analysis of PARK16}

SHEsis (http://analysis.bio-x.cn) analysis was performed to construct the haplotypes, as shown in Table 3. The G-A haplotype was very common in the case group compared to the control group $(\mathrm{P}<0.01 ; \mathrm{OR}=1.82,95 \%$ confidence interval: 1.44-2.30). However, the frequency of the A-A haplotype was higher in the control group than in the case group $(\mathrm{P}<0.01$; $\mathrm{OR}=0.48,95 \%$ confidence interval: 0.36-0.66). 


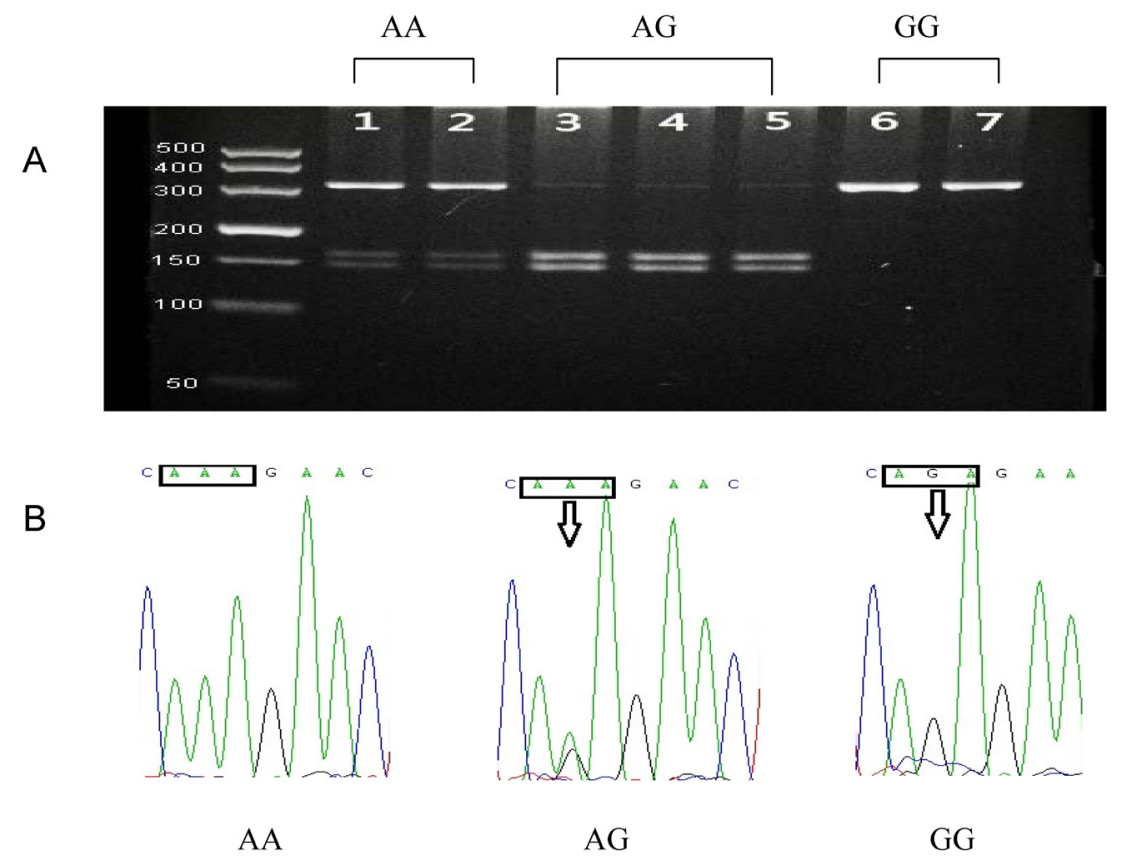

Figure 1. Genotyping results of rs947211. A. Result of RFLP. B. Result of sequencing.

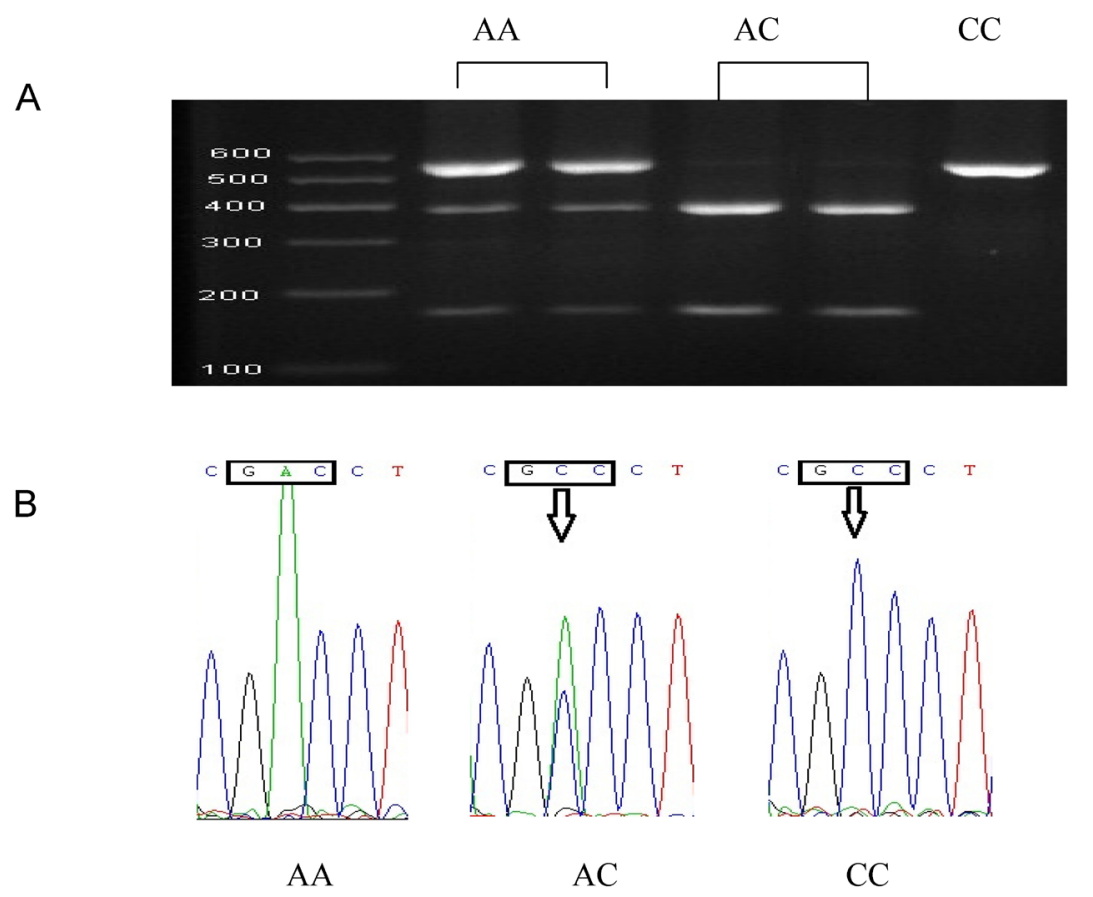

Figure 2. Genotyping results of rs823144. A. Result of RFLP. B. Result of sequencing. 


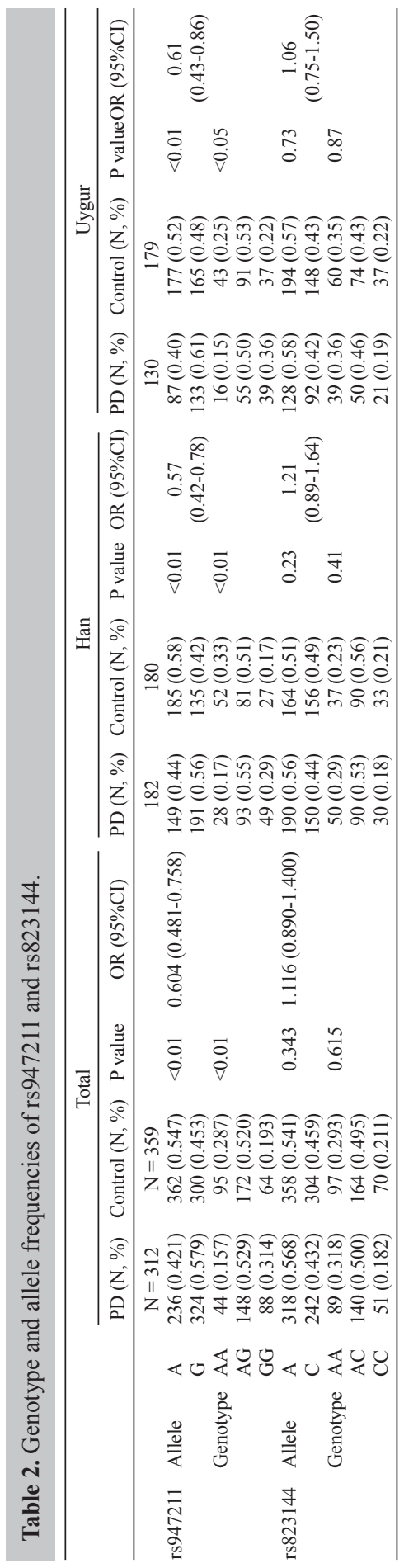




Table 3. Haplotype analysis.
\begin{tabular}{lcrrrrr}
\hline SNPs & Haplotype & PD $(\%)$ & Control $(\%)$ & $\chi^{2}$ & P value & OR (95\%CI) \\
\hline rs947211-rs823144 & A-A & $69.48(0.12)$ & $149.15(0.23)$ & 21.16 & $<0.01$ & $0.48(0.36-0.66)$ \\
& A-C & $166.52(0.30)$ & $212.85(0.32)$ & 0.83 & 0.36 & $0.89(0.70-1.14)$ \\
& G-A & $248.52(0.44)$ & $201.85(0.31)$ & 25.14 & $<0.01$ & $1.82(1.44-2.30)$ \\
& G-C & $75.48(0.14)$ & $98.15(0.15)$ & 0.45 & 0.5 & $0.90(0.65-1.24)$ \\
\hline
\end{tabular}

\section{DISCUSSION}

Satake et al. (2009) found that rs 947211 showed the most significant association with PD. This polymorphism is located $8.5 \mathrm{~kb}$ upstream of the $R A B 7 L 1$ gene and $5.6 \mathrm{~kb}$ downstream of the SLC41A1 gene in PARK16. However, linkage disequilibrium analysis showed that the locus was closely associated with NUSKS1 transcript levels, which encodes a nuclear protein containing common phosphorylation sites of casein kinase II and cyclin-dependent kinase (Ostvold et al., 2001). When we explored the association of rs 947211 in the PARK16 gene with PD in Uygur and Han Chinese subjects in Xinjiang, we found that individuals with the A allele had a significantly lower risk of suffering from $\mathrm{PD}$ than individuals carrying the $\mathrm{G}$ allele $(\mathrm{P}<0.01, \mathrm{OR}=0.60)$. Our results were consistent with several studies in Asian population (Satake et al., 2009; Tan et al., 2010). However, similar studies including Europeans showed that this locus was not associated with the occurrence of PD (Ramirez et al., 2011; Mata et al., 2011), further suggesting that the presence of polymorphic loci differs among various races.

The rs 823144 locus is located in the $R A B 7 L 1$ promoter region, and full-length $R A B 7 L 1$ cDNA encodes the RAB7 protein, which contains 203 amino acids. RAB7 is a member of the renin-angiotensin system oncogene family. More than 60 members of this family are considered to play an important role in regulating material exchange on both sides of the membrane through protein structure (Shimizu et al., 1997; Verhoeven et al., 2003). Although our results showed that the polymorphism was not associated with PD in Uygur and Han subjects in Xinjiang, Gan-Or et al. (2012) used the online software TFSEARCH to predict its association with $\mathrm{PD}$ and found that the polymorphism may exclude the binding sites of transcription factor cEts- 1 and increase binding to 3 other sites (p300, GATA-1, and Sp1), altering PD susceptibility between individuals. MacLeod et al. (2013) found that the LRRK2 (G2019S) mutation drastically reduced neurite length in rats. Overexpression of $R A B 7 L 1$ can suppress this phenomenon, suggesting that the gene's function may be related to the pathogenesis of PD caused by LRRK2 mutations. Our haplotype results showed that 2 SNPs had weak lineage, and that the A-A combination may be a protective factor against Parkinson's disease $(\mathrm{P}<0.05, \mathrm{OR}=0.36-0.66)$. The G-A combination significantly increased the risk of Parkinson's disease $(\mathrm{P}<0.05,1.44-2.30)$.

In summary, we verified that rs947211 was associated with PD in the Uygur and Han Chinese populations in Xinjiang. Although the results showed that rs823144 was not associated with PD, analysis of a larger sample study is necessary.

\section{REFERENCES}

Chang XL, Mao XY, Li HH, Zhang JH, et al. (2011). Association of GWAS loci with PD in China. Am. J. Med. Genet. B Neuropsychiatr. Genet. 156B: 334-339.

Chiang HL, Lee-Chen GJ, Chen CM, Chen YC, et al. (2012). Genetic analysis of HLA-DRA region variation in Taiwanese Parkinson's disease. Parkinsonism Relat. Disord. 18: 391-393. 
Gan-Or Z, Bar-Shira A, Dahary D, Mirelman A, et al. (2012). Association of sequence alterations in the putative promoter of RAB7L1 with a reduced parkinson disease risk. Arch. Neurol. 69: 105-110.

Goedert M, Spillantini MG, Del Tredici K and Braak H (2012). 100 years of Lewy pathology. Nat. Rev. Neurol. 9: 13-24. Lang AE and Lozano AM (1998). Parkinson's disease. First of two parts. N. Engl. J. Med. 339: 1044-1053.

Liu X, Cheng R, Verbitsky M, Kisselev S, et al. (2011). Genome-wide association study identifies candidate genes for Parkinson's disease in an Ashkenazi Jewish population. BMC Med. Genet. 12: 104.

Mata IF, Yearout D, Alvarez V, Coto E, et al. (2011). Replication of MAPT and SNCA, but not PARK16e18, as susceptibility genes for Parkinson's disease. Mov. Disord. 26: 819-823.

MacLeod DA, Rhinn H, Kuwahara T, Zolin A, et al. (2013). RAB7L1 interacts with LRRK2 to modify intraneuronal protein sorting and Parkinson's disease risk. Neuron 77: 425-439.

Ostvold AC, Norum JH, Mathiesen S, Wanvik B, et al. (2001). Molecular cloning of a mammalian nuclear phosphoprotein NUCKS, which serves as a substrate for Cdk1 in vivo. Eur. J. Biochem. 268: 2430-2440.

Ramirez A, Ziegler A, Winkler S, Kottwitz J, et al. (2011). Association of Parkinson disease to PARK16 in a Chilean sample. Parkinsonism Relat. Disord. 17: 70-71.

Satake W, Nakabayashi, Y, Mizuta I, Hirota Y, et al. (2009). Genome-wide association study identifies common variants at four loci as genetic risk factors for Parkinson's disease. Nat. Genet. 41: 1303-1307.

Shimizu F, Katagiri T, Suzuki M, Watanabe TK, et al. (1997). Cloning and chromosome assignment to 1q32 of a human cDNA (RAB7L1) encoding a small GTP-binding protein, a member of the RAS superfamily. Cytogenet. Cell Genet. 77: 261-263.

Simón-Sánchez J, van Hilten JJ, van de Warrenburg B, Post B, et al. (2011). Genome-wide association study confirms extant PD risk loci among the Dutch. Eur. J. Hum. Genet. 19: 655-661.

Tan EK, Kwok HH, Tan LC, Zhao WT, et al. (2010). Analysis of GWAS-linked loci in Parkinson disease reaffirms PARK16 as a susceptibility locus. Neurology 75 : 508-512.

Tucci A, Nalls MA, Houlden H, Revesz T, et al. (2010). Genetic variability at the PARK16 locus. Eur. J. Hum. Genet. 18: 1356-1359.

Verhoeven K, De Jonghe P, Coen K, Verpoorten N, et al. (2003). Mutations in the small GTP-ase late endosomal protein RAB7 cause Charcot-Marie-Tooth type 2B neuropathy. Am. J. Hum. Genet. 72: 722-727.

Vilariño-Guell C, Ross OA, Aasly JO, White LR, et al. (2010). An independent replication of PARK16 in Asian samples. Neurology 75: 2248-2249.

Yan YP, Mo XY, Tian J, Zhao GH, et al. (2011). An association between the PARK16 locus and Parkinson's disease in a cohort from eastern China. Parkinsonism Relat. Disord. 17: 737-739. 